\title{
NUEVAS ESPECIES DE CARABODIDAE (ACARIFORMES, ORIBATIDA) IBEROMAGREBÍES
}

\author{
L. S. Subías (*) y A. Arillo (*)
}

\begin{abstract}
RESUMEN
El presente trabajo está dedicado al estudio de varias especies de Carabódidos iberomagrebíes; cuatro de ellas son nuevas para la ciencia: Carabodes pirenaicus sp. nov., Carabodes guadarramicus sp. nov., Odontocepheus bandae sp. nov. y Odontocepheus zaballosi sp. nov.; las dos primeras proceden de España, mientras que las otras dos son marroquíes; también se redescribe y aportan nuevos datos sobre la especie mediterráneo-occidental Carabodes quadrangulus Bernini, 1979.
\end{abstract}

Palabras clave: Acariformes, Oribatida, Carabodidae, Taxonomía, Nuevas especies, Carabodes, Odontocepheus, España, Marruecos.

\section{ABSTRACT \\ New iberomagrebian species of Carabodidae (Acariformes, Oribatida)}

In this paper five species belonging to the family Carabodidae are studied; four of them are new species: Carabodes pirenaicus sp. nov. and Carabodes guadarramicus sp. nov. from Spain, and Odontocepheus bandae sp. nov. and Odontocepheus zaballosi sp. nov. from Morocco; Carabodes quadrangulus Bernini, 1979, a west-mediterranean species, is redescribed and commented on.

Key words: Acariformes, Oribatida, Carabodidae, Taxonomy, New species, Carabodes, Odontocepheus, Spain, Morocco.

\section{Introducción}

La familia Carabodidae C. L. Koch, 1837 está muy bien representada en el Mediterráneo Occidental; según Subías y Gil-Martín (1997) serían 37 las especies válidas conocidas hasta la fecha en dicha región, la mayoría pertenecientes al género Carabodes C. L. Koch, 1835, del que se van a describir en el presente trabajo dos nuevas especies, procedentes de España, aportándose nuevos datos sobre otra interesante especie mediterráneo-occidental. Por lo que respecta al género Odontoce- pheus Berlese, 1913, son 7 las especies conocidas hasta ahora en el área de estudio; si tenemos en cuenta que sólo se conocen 10 especies en todo el mundo, se podría concluir que el Mediterráneo Occidental sería su centro de origen y dispersión (Baratti \& Bernini, 1994), lo que vendría a corroborarse con la descripción en el presente trabajo de dos nuevas especies más procedentes de Marruecos.

Todo el material tipo se encuentra depositado en la Cátedra de Entomología del Departamento de Zoología de la Universidad Complutense de

* Departamento de Zoología. Facultad de Biología. Universidad Complutense. 28040 Madrid. E-mail: subias@eucmax.sim.ucm.es_aarillo@teleline.es 
Madrid, bien conservado en ácido láctico al 70\% o bien montado en preparaciones microscópicas con "Hoyer".

\section{Carabodes pirenaicus sp. nov. (Figs. 1-3)}

Material TíPICO: Se han estudiado dos ejemplares, machos ambos, de los que uno ha sido designado como holotipo y el otro sería el paratipo, encontrándose ambos conservados en ácido láctico al 70\%. Proceden de los Pirineos, de la localidad de Bono (Lérida), y han sido recolectados de una muestra de hojarasca y musgo, en un bosque mixto de hayas y abetos, por L. S. Subías el día 29 de agosto de 1983.

DIMENSIONES Y TEGUMENTO: El tamaño de los dos ejemplares es muy similar ya que su longitud es de $510 \mu \mathrm{m}$ y $520 \mu \mathrm{m}$ y su anchura de $330 \mu \mathrm{m}$ en ambos.

Están bien esclerotizados y su coloración es castaño-rojiza no demasiado oscura. Todo el cuerpo está cubierto por una delgada capa cerotegumentaria, obserbable en los contornos del cuerpo y que en algunas partes parece desprenderse algo tras el calentamiento con el ácido láctico para su aclarado.

PRODORSO: Rostro redondeado y setas rostrales lisas y arqueadas hacia el interior, mientras que las setas lamelares, insertas en el exterior de las cúspides lamelares, son bastante más robustas y con una barbulación (más bien formada por espinas) externa, mostrando también una fuerte curvatura hacia el interior del prodorso; las setas interlamelares se dirigen hacia arriba y un poco hacia el interior y son robustas, dilatándose algo hacia el extremo barbulado; se insertan sobre la base interna de las lamelas, en la mitad de la zona donde se originan sendas elevaciones que atraviesan el prodorso posteriormente, a modo de puente tuberculado, ya que se unen centralmente, y por detrás del cual existe un amplio espacio menos esclerotizado, a modo de "cuello" o cavidad cervical, que separa el prodorso del notogáster y que presenta un fino y denso punteado. Por lo que respecta a la escultura del resto de prodorso, las lamelas y la regiòn rostral muestran un foveolado regular, también presente en forma de sendas hileras longitudinales en la zona prodorsal junto a la inserción de las lamelas. Los botridios son grandes y se abren lateralmente en los ángulos posteriores del prodorso (por delante de ellos se aprecian los grandes tectopedios del primer par de patas), y los sensilos son de tallo de longitud moderada y cuyo extremo, que se arquea hacia arriba, se dilata por la presencia de un "cepillo" de bárbulas (más bien papilas) dispuestas en su cara externa.
NOTOGÁster: Ancho y convexo, con un surco circunnotogastral que lo bordea completamente lateral y posteriormente, delimitando una franja externa que presenta un denso y uniforme tuberculado, mientras que la escultura del resto del notogáster está contituida por una densa y prieta serie de tubérculos aplanados y de bordes angulosos, densamente punteados, que le confieren un aspecto reticulado-poligonal. El borde anterior del notogáster presenta una patente protuberancia o apófisis central y dos más pequeñas y menos perceptibles a los lados de ésta; en los bordes anterolaterales se aprecian bien desarrollados sendos salientes o láminas humerales, angulosas posteriormente y que anteriormente contactan con los botridios. Las setas notogastrales, en su número habitual de 10 pares, son de longitud media, algo arqueadas, robustas y con una parte distal con varias papilas o espinosidades alineadas longitudinalmente en su borde superior externo; el par delantero anterior (las setas $c 2$ ) se encuentra alineado con la hilera interior de setas $(l m, l p, h 1$ y $p 1)$.

REGIÓN VENTRAL: En la regiòn epimeral tanto los epímeros como los apodemas que los separan están bien marcados, uniéndose estos últimos centralmente en una ancha banda longitudinal lisa desprovista de escultura alguna, ya que los epímeros muestran un foveolado regular muy nítido, similar al que también muestra el mentun del gnatosoma. Las placas genitales y anales también presentan foveolado, aunque las foveolas son mucho menores, pareciendo casi más un punteado grueso; en cambio la placa ventral muestra una escultura de retículo de tubérculos punteados, muy similar a la notogastral, excepto la región que rodea a las placas anales que sólo presenta un denso punteado. Respecto a la quetotaxia de la región epimeral es la típica (3-1-3-3) y está constituida por setas lisas y finas, de diferente longitud, siendo las $1 a$ y $2 a$ las menores, muy cortas, mientras que las $4 c$ son las más largas; entre las inserciones de las patas III y IV aparecen unos prominentes discidios bastante estrechos y agudos. Los cuatro pares de setas genitales son largas, finas y lisas; se disponen a una distancia regular unas de otras en el borde interno de cada placa y se dirigen todas hacia atrás; el par de setas adgenitales, también largas como las anteriores, se curva hacia dentro; por otra parte los dos pares de setas anales son bastante más cortos y muy similares al par de setas adanales $a d 3$; en cambio las setas adanales $a d 1$ y $a d 2$ son mayores, similares a las notogastrales, aunque más cortas y menos robustas. 


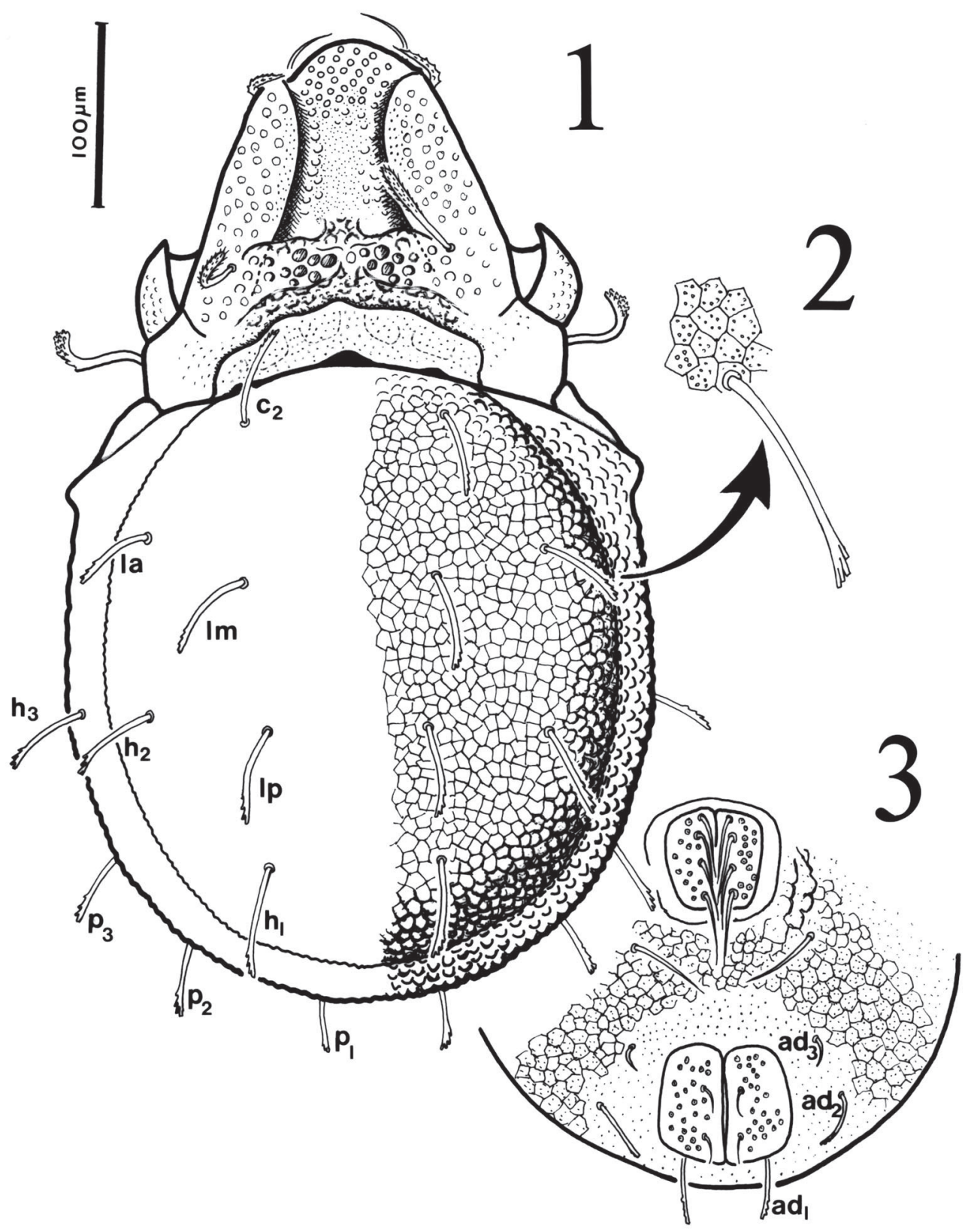

Figs. 1-3.-Carabodes pirenaicus sp. nov. 1) Visión dorsal del cuerpo. 2) Detalle de la escultura y seta notogastrales. 3) Placa ventral.

Figs. 1-3.- Carabodes pirenaicus sp. nov. 1) Body dorsal view. 2) Detail of notogastral sculpture and seta. 3) Ventral plate.

PATAS: Todas las patas son monodáctilas, con los trocánteres y fémures más robustos que el resto de segmentos, pudiéndose replegar a los lados del cuerpo.
AfINIDADES: Siguiendo el criterio de Baratti y Bernini (1996) pertenecería al grupo de especies denominado "marginatus", caracterizado por la presencia de una escultura notogastral tuberculado- 
reticulada y una amplia cavidad cervical entre el prodorso y el notogáster, y en el que se incluirían, aparte de Carabodes marginatus (Michael, 1884) (= Carabodes pontiger Berlese, 1913), las especies Carabodes quadrangulus Bernini, 1979 (que se redescribirá a continuación), Carabodes chirstlus Mahunka, 1987 (= Carabodes foliatus Morell, 1990) y Carabodes montanus Bernini, 1979 (especie descrita sólamente al microscopio electrónico), muy similar a las anteriores, pero que no es mencionada por Baratti y Bernini (op. cit.) a pesar de haber sido descrita por Bernini (1979) en el mismo trabajo que C. quadrangulus. Las tres últimas especies presentan en la parte posterior del prodorso dos grandes protuberancias tuberculadas bien separadas una de otra y los sensilos son más alargados y puntiagudos; en cambio en C. marginatus y en la nueva especie dichas protuberancias se encuentran unidas centralmente formando una especie de puente y los sensilos son algo más dilartados distalmente, presentando además $C$. chirstlus las setas notogastrales más ensanchadas, casi foliáceas. Por lo tanto $C$. pirenaicus a la especie que más se asemeja de las mencionadas es a C. marginatus, pero sus setas notogastrales son más largas y robustas, el tuberculado de la placa ventral es como el notogastral, reticulado, en cambio en $C$. marginatus los tubérculos están bien separados entre sí, no formando ningún retículo; además la nueva especie presenta una ancha banda longitudinal central lisa entre los epímeros, carácter este que no presenta ninguna de las otras especies mencionadas que muestran dicha región también foveolada, como los epímeros. Tampoco, salvo C. quadrangulus, ninguna de las especies del grupo "marginatus" presenta la destacada apófisis central del borde anterior del notogáster. De todas formas a quien más se asemeja $C$. pirenaicus es a la especie búlgara Carabodes magnus Kunst, 1961 (tampoco incluida en el grupo "marginatus" por Baratti y Bernini, op. cit.), pero sus dimensiones son mucho mayores (900-1.020 $\mu \mathrm{m}$ x 570-624 $\mu \mathrm{m}$ según Kunst, 1961) y el reticulado de la placa ventral parece distinto al del notogáster.

Biología: Los ejemplares estudiados proceden de hojarasca y musgo de un hayedo mixto con abetos. Es muy probable que los individuos descritos por Moraza et al. (1980), bajo la denominación de Carabodes reticulatus Berlese, 1913, sean idénticos a los aquí descritos como $C$. pirenaicus ya que en la redescripción que dan los autores los representan con una escultura notogastral tuberculadopoligonal punteada, así como con la apófisis central del borde anterior del notogáster patente, lo que parece confirmar dicha identidad. Además dichos ejemplares proceden también de una muestra de hojarasca recolectada en un hayedo de los Pirineos occidentales, lo que corroboraría la preferencia por los hayedos pirenaicos de la nueva especie.

Etimología: Por parecer restringida, al menos por el momento, la distribución de esta nueva especie a los Pirineos, se le ha asignado la denominación específica de "pirenaicus".

\section{Carabodes quadrangulus s. l. Bernini, 1979 (Fig. 4)}

MATERIAL ESTUDIADO: Los ejemplares utilizados para este estudio han sido tres, dos machos y una hembra, procedentes de Torrelaguna (Madrid) y recolectados el día 9 de marzo de 1987 en suelo bajo un enebro, en una zona quemada dos años antes, por A. Ajo. Dichos ejemplares se encuentran montados en una preparación microscópica con "Hoyer".

Discusión: Especie parecida a la anterior, pero en la que desde un principio nos llamó la atención la presencia de sensilos largos y setiformes, algo infrecuente dentro del género Carabodes. Por otra parte, su peculiar escultura notogastral constituida por tubérculos poligonales y demás características morfológicas la aproximaban extraordinariamente a la especie C. quadrangulus, especie de distribución básicamente mediterráneo-occidental, pero que en la descripción de Bernini (1979) estaría dotada de un sensilo cuya barbulación en el extremo le darían a éste la configuración de un mayor o menor ensanchamiento, como en los ejemplares ya citados anteriormente en España. Pero, por un lado, el hecho de que el mismo Bernini (op. cit.) comenta la variabilidad existente en los ejemplares por él estudados a este respecto, y por otra parte el hecho de que se nos han dado los dos tipos de sensilos simultáneamente en un ejemplar, nos lleva a concluir que entran dentro de la variabilidad de dicha especie. Lo mismo se podría decir respecto a la apófisis central del borde del notogáster que presentan nuestros ejemplares (aparte de las mucho menos desarrolladas laterales) que, aunque no mencionadas por dicho autor en la descripción original, sí que parece intuirse en una de las fotografías al microscopio electrónico que acompaña al trabajo. En lo que sí encajan con dichos ejemplares es en las dimensiones que presentan los nuestros (500$515 \mu \mathrm{m}$ de longitud por $300-310 \mu \mathrm{m}$ de anchura).

También existe otra especie descrita por Mihelcic (1966) de Madrid, Carabodes flagellifer, 


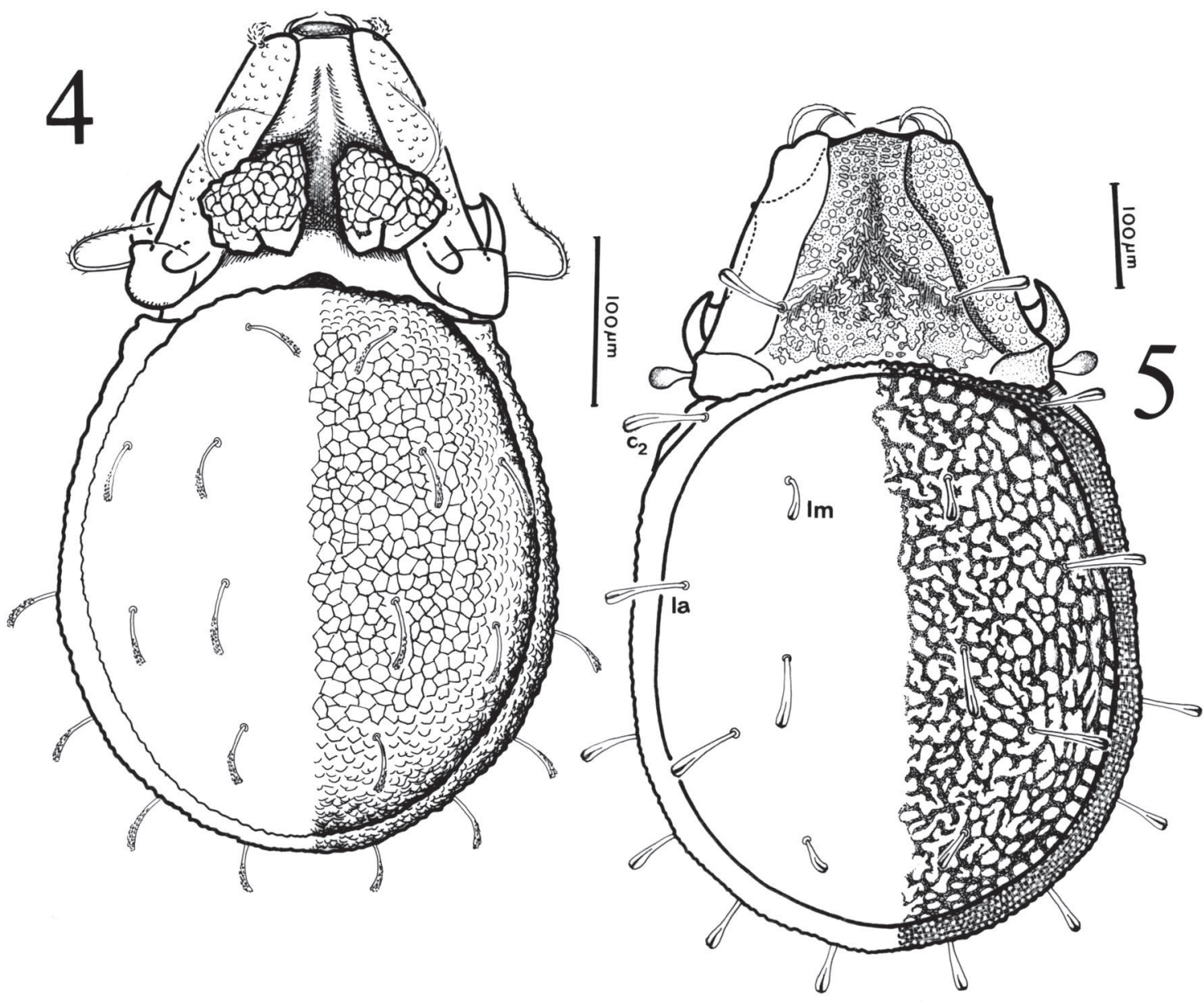

Figs. 4-5.- 4) Carabodes quadrangulus Bernini, 1979. Visión dorsal del cuerpo. 5) Carabodes guadarramicus sp. nov. Visión dorsal del cuerpo.

Figs. 4-5.- 4) Carabodes quadrangulus Bernini, 1979. Body dorsal view. 5) Carabodes guadarramicus sp. nov. Body dorsal view.

que presenta sensilos setiformes y la apófisis central del borde notogastral bien desarrollada y que PérezÍñigo (1997) considera species inquirienda ya que han desaparecido los ejemplares que sirvieron para su descripción. De todas formas, y aunque las descripciones de dicho autor no son muy fiables, la escultura del prodorso y la notogastral (tuberculado aparentemente no poligonal) parecen diferenciarla bien del auténtico $C$. quadrangulus que, por el momento, ha de considerarse como una "gran especie" con multitud de formas, tal vez subespecies, que el tiempo y el estudio de más ejemplares de distintas procedencias podrá dilucidar.
Carabodes guadarramicus sp. nov. (Fig. 5)

MATERIAL TÍPICO: Se han estudiado 8 ejemplares ( 5 hembras y 3 machos) procedentes del Puerto de la Fuenfría, en la Sierra de Guadarrama (Madrid), recolectados por L. S. Subías el día 11 de abril de 1975 en musgos y madera descompuesta de un tejo casi seco, a $1.500 \mathrm{~m}$ de altura. Tanto el holotipo (un macho) como los 7 paratipos se encuetran conservados en una preparación microscópica montados en "Hoyer".

DiMENSIONES Y TEGUMENTO: De gran tamaño, su longitud oscila entre los 760 y $950 \mu \mathrm{m}$ y su anchura entre los 475 y $610 \mu \mathrm{m}$, siendo el tamaño medio de las hembras algo mayor que el de los machos. 
El tegumento está muy esclerotizado y bien esculpido (escultura que se describirá más detalladamente en cada apartado morfológico), dando a los individuos una coloración castaño-rojiza oscura, negruzca. Todo el cuerpo parece cubierto por una delgada membrana cerotegumentaria que en algunas partes aparece desprendida.

PRODORSO: Tanto las lamelas como el prodorso presentan un nítido foveolado que en la parte central posterior del mismo tiende a ser más irregular por fusión de unas foveolas con otras dando lugar a sendas quillas que convergen hacia delante en una quilla central que casi alcanza el borde rostral y que da lugar a sendas depresiones a los lados interiores de cada lamela. El rostro es redondeado y las setas rostrales robustas, algo ásperas externamente y curvadas hacia dentro; las lamelas alcanzan el borde rostral y las setas lamelares se insertan cerca de los bordes externos de sus cúspides; son similares a las rostrales, aunque algo mayores. En visión dorsal, y por transparencia, se puede observar una región más clara en la zona distal de dichas lamelas que coincide con la parte donde acaban los tutorios, que son prominentes, robustos y de extremo algo denticulado. Las setas interlamelares son erectas, muy parecidas a las notogastrales, y se insertan por delante de los botridios, junto al borde interno lamelar y en la zona donde comienzan las quillas prodorsales. A los lados de la base de las lamelas se observan los tectopecios del primer par de patas, y por detrás de ellas se sitúan los botridios, cuya abertura es lateral y de los que salen sendos sensilos que son muy cortos y globosos, presentando la parte distal de la cabeza cubierta de diminutas papilas. El prodorso se puede considerar "sentado" sobre el notogáster, no existiendo, como en los Carabodes anteriormente descritos, ningún amplio espacio cervical que los separe.

NotogÁster: Ancho, convexo y recorrido en todo su contorno exterior por un surco circunnotogastral que en sus bordes laterales anteriores originan sendas prominencias o salientes humerales redondeados que contactan con el borde posterior de los botridios. La escultura del notogáster está constituidas por amplias foveolas irregulares y coalescentes unas con otras confiriéndole un aspecto laberíntico al mismo, salvo en la región externa delimitada por el surco circunnotogastral que presenta un denso y regular tuberculado. Los 10 pares de setas notogastrales son muy similares entre sí, de longitud moderada, robustas y que se van ensanchando hacia el extremo, dando lugar a una estre- cha "espátula" microbarbulada, presentando un eje longitudinal central que llega desde el extremo hasta aproximadamente la mitad de la seta; el par de setas anterior (las c2) están muy desplazadas hacia los bordes, por lo que se alinean con las setas de la hilera media (la, $h 2$ y $p 2$ ).

REGIÓN VENTRAL: La región epimeral presenta un foveolado-reticulado bastante uniforme, mientras que el mentun, placas genitales y placas anales están regularmente foveolados y dichas foveolas son menores. Los epímeros y apodemas están bien marcados y la quetotaxia de la región epimeral es la habitual (3-1-3-3) presentando sus setas de diferente longitud, pero más bien cortas, finas y lisas siendo las $1 a, 2 a$ y $3 a$ casi imperceptibles; los discidios son prominentes y puntiagudos. La escultura de la placa ventral es parecida a la notogastral, de aspecto laberíntico todavía más marcado. Las placas genitales portan cuatro pares de setas regularmente dispuestas en su borde interno; son relativamente largas, finas y lisas, y de ellas los tres primeros pares se dirigen hacia atrás, mientras que el cuarto se dirige hacia delante (cuando lo habitual es que se dirijan todos hacia atrás); los dos pares de setas anales son más cortos. El par de setas adgenitales está bien desarrollado, como las genitales, mientras que las adanales ad3 son más similares a las anales; en cambio las adanales ad1 y ad2 son similares a las notogastrales, aunque de menor tamaño.

PATAS: Todas las patas son monodáctilas y presentan un pequeño diente interno cerca de su inserción, siendo las uñas del primer par de patas algo menores que las demás. Ventralmente, los fémures de las patas III y IV presentan un espolón distal que se observa muy bien en visión lateral. Todos los trocánteres y fémures son robustos y regularmente foveolados, mientras que el resto de las patas son delgadas y de tegumento liso, presentando las zonas laterales del prosoma adaptadas para que puedan replegarse alli las patas, seguramente en posición defensiva.

AfINIDADES: Por el tipo de escultura corporal y sensilo, y por no presentar "cuello" o espacio cervical entre el prodorso y el notogáster, la nueva especie se asemeja mucho a Carabodes labyrinthicus (Michael, 1879) (especie holártica según Subías y Gil-Martín, 1997), pero se diferencian claramente ambas especies porque las setas notogastrales de esta última son claramente setiformes, mientras que las de $C$. guadarramicus se van ensanchando hacia el extremo hasta adquirir un aspecto más o menos 
espatulado. En cambio este tipo de setas son muy semejantes a las que muestra la especie norteamericana Carabodes california Reeves y BehanPelletier, 1998; en cambio la escultura tuberculada notogastral y el sensilo alargado son muy diferentes de los de la nueva especie. También se asemeja mucho a la especie japonesa Carabodes breviclava Aoki, 1970, pero sus setas notogastrales, aunque ensanchadas hacia el extremo, no terminan espatuladas sino que presentan dicho extremo unilateralmente barbulado por su cara externa.

Biología: Los ejemplares utilizados para la descripción de esta nueva especie han sido recolectados en la base de un tronco (de tejo) donde había musgos y madera en descomposición; pero es que también se han recolectado algunos otros ejemplares en otra localidad de la Sierra de Guadarrama, en El Escorial a $1.000 \mathrm{~m}$ de altura, también en musgos y corteza descompuesta (en este caso de la base de un roble), por lo que parece ser dicho medio el preferido por esta especie.

Etimología: El nombre específico de "guadarramicus" dado a esta especie hace referencia al macizo montañoso, Sierra de Guadarrama, donde parece estar, por el momento, restringida su distribución, no siendo de extrañar que en un grupo con tan elevado grado de especiación en el Mediterráneo Occidental aparezcan especies endémicas de los distintos macizos montañosos (como es también el caso de la especie C. pirenaicus, descrita previamente).

\section{Odontocepheus bandae sp. nov. (Figs. 6 y 7)}

MATERIAL TíPICO: Sólo se ha recolectado un ejemplar de esta especie, el holotipo, pero que es tan diferente a los demás Odontocepheus que sin lugar a dudas se trata de una nueva especie completamente diferente a las conocidas actualmente. Se trata de una hembra procedente de Marruecos (al norte de Meknés), recolectada por J. P. Zaballos y E. Banda el día 20 de febrero de 1999 en la ladera (cardonal con palmitos) de un campo de cultivo. Dicho ejemplar, una hembra, se encuentra conservado en una preparación microscópica con "Hoyer".

DimenSIONES Y TEGUMENTO: Su longitud es de $660 \mu \mathrm{m}$ y su anchura de $345 \mu \mathrm{m}$, sin tener en cuenta el grosor de la capa que recubre todo el cuerpo, sobre todo dorsalmente, y que está formada por partículas diversas del medio donde viven adheridas a su capa cerotegumentaria y que, junto con su capacidad de replegar la patas en las regiones laterales del cuerpo, deben de mimetizar tanto con el medio donde vive a este oribátido que le hace pasar, con toda seguridad, desapercibido a sus depredadores. Al intentar desprender dicha capa, sobre todo de gran espesor sobre el notogáster, para poder observar su morfología, se han desprendido con ella algunas setas corporales; con las del notogáster no hay problemas porque han quedado muchas $\mathrm{y}$ todas deben de ser muy similares entre sí, pero no hemos podido observar ninguna seta interlamelar, por lo que en el dibujo que acompañamos figuran representadas con trazos discotinuos como seguramente deberían de ser, ya que todas las especies de Odontocepheus conocidas las presentan siempre muy similares. El tegumento está bien esclerotizado y es de color pardo-rojizo oscuro.

PRODORSO: Setas rostrales y lamelares robustas y arqueadas, las primeras hacia arriba y las segundas (que se insertan en el borde externo de cada cúspide lamelar) hacia dentro. Las lamelas, anchas, largas y casi parlelas, como es habitual en el género, presentan algunas estrías transversales en su tercio distal, estando unidas anteriormente sus cúspides lamelares (que pueden llegar a sobrepasar el borde rostral) por una estrecha translamela, por detrás de la cual se observan sendas alineaciones de nítidas foveolas en la parte anterior del prodorso, situadas junto al borde interno de cada lamela; en la parte central del tercio posterior del prodorso aparece un tubérculo o apófisis impar dirigida hacia delante, mientras que en la parte posterior prodorsal, y entre los botridios, existen un par de robustas y bien esclerotizadas apófisis dirigidas hacia atrás. Botridios grandes, situados en los ángulos posteriores del prodorso y con sus aberturas laterales; los sensilos son atípicos, finos, setiformes, auqueados hacia dentro y con una hilera de cortas y distanciadas bárbulas en su borde externo. Tutorios dentados y tectopedios I muy prominentes (los tectopedios II no están tan desarrollados).

NotogÁster: Como es habitual en el género es alargado, algo ensanchado posteriormente, con la parte central convexa y bordeada por un surco cicunnotogastral, y con el borde anterior truncado; en dicho borde se sitúan un par de robustas y bien esclerotizadas apófisis enfrentadas a las prodorsales. Los ángulos humerales son prominentes, bien desarrollados y redondeados. La escultura notogastral (una vez retirada la cubierta de "camuflaje" que lo recubre) también es atípica en los Odontocepheus pues está formada por gruesos gránulos o tubérculos redondeados distribuidos regularmente y con cierta tendencia a disponerse en 


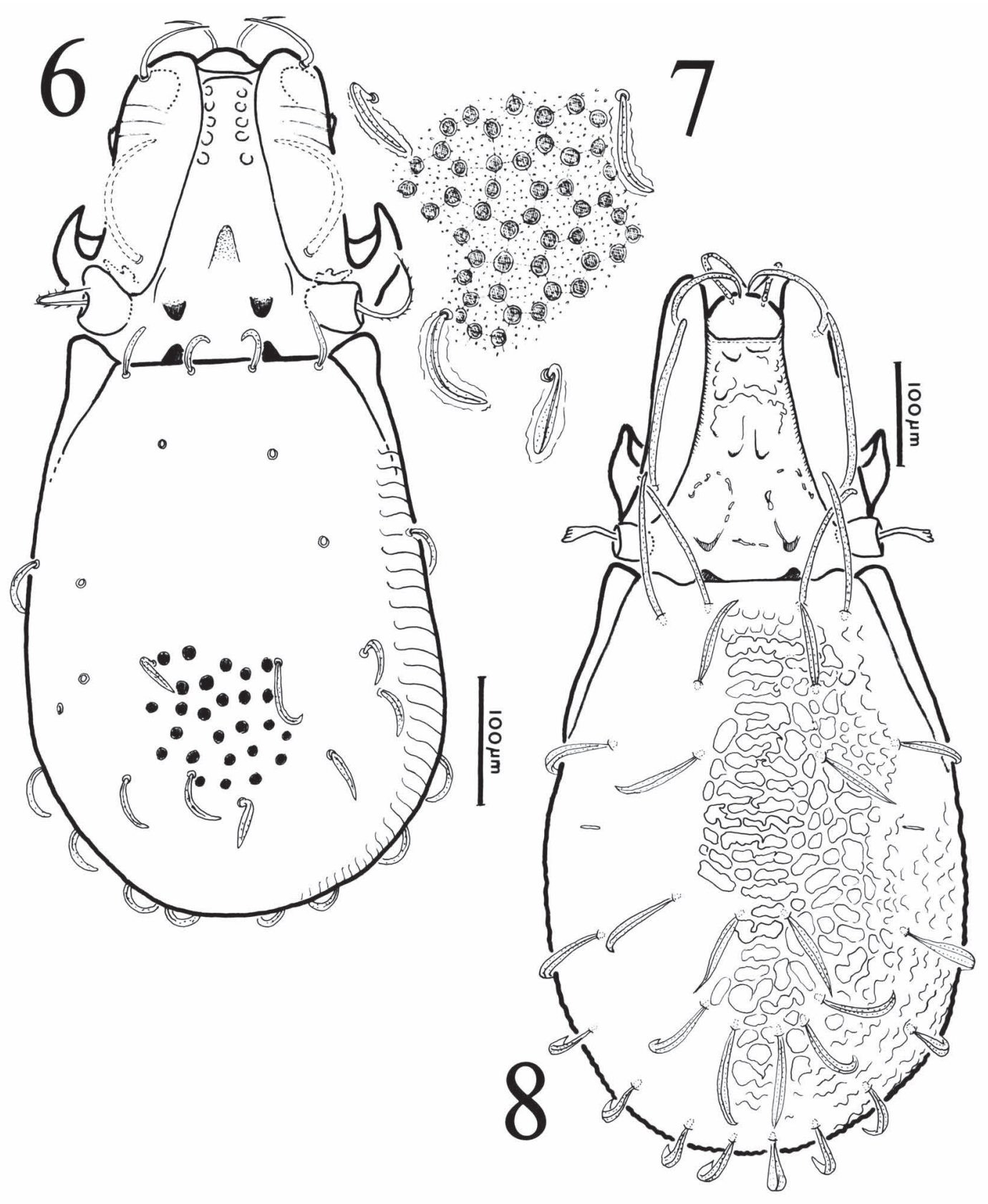

Figs. 6-8.- 6-7) Odontocepheus bandae sp. nov. 6) Visión dorsal del cuerpo. 7) Detalle de la escultura y setas notogastrales. 8) Odontocepheus zaballosi sp. nov. Visión dorsal del cuerpo.

Figs. 6-8.- 6-7) Odontocepheus bandae sp. nov. 6) Body dorsal view. 7) Detail of notogastral sculpture and setae. 8) Odontocepheus zaballosi sp. nov. Body dorsal view.

forma de "celdillas de panal", excepto en la parte externa, delimitada por el surco circunnotogastral, que presenta una sucesión regular de quillas o plie- gues transversales; el resto del notogáster muestra un punteado muy fino y denso. Los 14 pares de setas que tiene presentan la disposición típica, con 
los tres primeros pares $(c 1, c 2$ y probablemente también el $d a$ ) dirigidos hacia delante; todas las setas son de tamaño y forma similar, arqueadas, cortas, punteadas, robustas y lanceolado-foliáceas, con un eje central y un reborde hialino cerotegumentario.

REGIÓN VENTRAL: Todos los apodemas de la región epimeral están bien desarrollados, uniéndose centralmente en una banda longitudinal más oscura. Los epímeros están desprovistos de escultura y las setas epimerales son cortas y heterogéneas, con algunas ligeramente lanceoladas. La placa ventral presenta una escultura tuberculada similar a la notogastral, pero en este caso no es tan regular su distribución y muestran cierta tendencia a unirse unos tubérculos con otros. Las placas genitales son más pequeñas que las anales y distan bastante de ellas, presentando los cuatro pares de setas habituales del género dispuestas en su borde interno; dichas setas, al igual que las adgenitales son cortas y ligeramente lanceoladas; los dos pares de setas anales son muy cortos y finos, mientras que las adanales son similares a las notogastrales, aunque bastante más cortas.

PATAS: Todas las patas son mondáctilas y con los fémures de todas ellas y los trocánteres III y IV robustos y recubiertos por la capa cerotegumentario-detrítica, mientras que los segmentos distales de las patas, que son más delgados, están desprovistos de ella.

AfINIDADES: Por el tamaño y forma de las setas notogastrales se asemeja a la especie española Odontocepheus curtiseta Ruiz, Subías y Kahwash, 1989, pero se diferencia cláramente, tanto de ella como de los demás Odontocepheus, porque la nueva especie tiene los sensilos setiformes (en lugar de presentar extremos más o menos dilatados) y la escultura notogastral y de la placa ventral consiste en tubérculos redondeados (en lugar del retículo-foveolado de las demás).

BiologíA: Por el lugar donde se ha recolectado, una ladera con cardos, palmitos y otra vegetación rala al lado de un campo de cultivo, no cabe duda de que se trata de una especie de tendencias cláramente xerófilas.

Etimología: El epíteto específico de "bandae" hace referencia a uno de los recolectores de la muestra de suelo donde ha aparecido esta especie, doña Eva Banda, a quien mostramos nuestro agradecimiento.

\section{Odontocepheus zaballosi sp. nov. (Fig. 8)}

Material tíPICO: Se ha recolectado un individuo hembra, el holotipo, en una muestra, procedente también de Marruecos (Jerada), recolectada por M. A. Ferrández el día 23 de abril de 1984 y constituida por hojarasca y suelo recolectados bajo un matorral de Pistacea, figurando como Odontocepheus sp. en el listado de oribátidos que de Marruecos y Sahara Occidental dan Subías et al. (1994). Dicho ejemplar se encuentra conservado en un tubo con ácido láctico al $70 \%$.

DimENSIONES Y TEGUMENTO: Cuerpo de grandes dimensiones, $820 \mu \mathrm{m}$ de longitud por $410 \mu \mathrm{m}$ de anchura, y alargado ya que, como se puede observar, es el doble de largo que de ancho.

Bien esclerotizado, aunque su coloración no es demasiado oscura. Todo el cuerpo y patas se encuentra cubierto de una fina capa cerotegumentaria.

PRODORSO: Rostro redondeado, con las setas rostrales robustas, largas y recurvadas hacia arriba y atrás, llegándose a tocas sus extremos. Las lamelas son anchas, largas y lisas, bastante paralelas entre sí y con sus cúspides, que se van estrechando hacia el extremo y pueden llegar a sobrepasar el borde rostral, estando unidas por una línea translamelar bien marcada. Las setas lamelares se insertan sobre las cúspides de cada lamela, en su parte central y a la altura de dicha línea translamelar; estas setas son muy similares a las rostrales, pero en este caso se recurvan hacia delante e internamente, llegándose a tocar también sus extremos, que a su vez contactan con los de las setas rostrales. Las setas interlamelares son como unas gruesas y largas cerdas que se insertan en las lamelas, cerca de sus bases, es decir, próximas y por delante de los botridios, y discurren hacia delante sobre dichas lamelas, sobrepasando algo las bases de sus cúspides. La escultura prodorsal no está demasiado marcada y consiste en una corta banda longitudinal central interlamelar que por delante acaba en otra banda, transversal convexa, por detrás de la línea translamelar; los bordes de dichas bandas están poco marcados y constituidos por una sucesión irregular de concavidades. En la parte posterior del prodorso, y junto a las bases lamelares, destacan algo más un par de elevaciones, laxamente tuberculadas, que posteriormente acaban en sendos cóndilos o apófisis, dirigidos hacia atrás, bien esclerotizados. Los botridios se disponen y abren posterolateralmente y de ellos salen sendos sensilos de tallo corto que se ensancha distalmente en forma de pequeña espátula cuya superficie está cubiereta de microespículas, las cuales recubren también todas las setas prodorsales mencionadas previamente. Por fuera de las 
bases lamelares se observan los tectopedios de las patas I bien desarrollados.

Notogáster: En el borde anterior, recto, se observan centralmente un par de apófisis esclerotizadas enfrentadas a las ya mencionadas de la parte posterior del prodorso, mientras que a cada lado presenta sendos salientes humerales, enfrentados a los botridios, que se prolongan hacia atrás en forma de estrecha lámina que se va atenuando progresivamente a lo largo del primer tercio del borde lateral notogastral. A los lados y posteriormente el notogáster presenta un depresión, o surco circunnotogastral, que hace que la parte central del notogáster se muestre claramente elevada o convexa; la escultura de esta parte central está constituida por un retículo formado por foveolas irregulares, más o menos rectangulares y dispuestas en bandas transversales, mientras que en el resto del notogáster estas foveolas, de contorno irregular, tienden a ser más redondeadas o poligonales. De los 14 pares de setas notogastrales presentes, los dos primeros (los $c 1$ y $c 2$ ) son como gruesas cerdas de aspecto similar a las setas interlamelares, aunque algo menos largas, y se dirigen hacia delante, sobre el prodorso, mientras que las restantes setas, algo más cortas, presentan un aspecto diferente ya que son lanceolado-foliáceas y arqueadas, con un eje central bien patente que las recorre en toda su longitud, estando también el par delantero central (las $d a$ ) dirigidas hacia delante; al igual que las setas prodorsales, también todas las setas notogastrales presentan su superficie completamente cubierta de diminutas microespículas.

REGIÓN VENTRAL: En la parte anterior, a los lados del camerostoma, se observan claramente una agrupación de espinas digitiformes que se corresponden con los extremos distales de cada uno de los tutorios. En la región epimeral, que es lisa, los apodemas están bien marcados y, por lo tanto, los epímeros bien delimitados; la fórmula quetotáxica epimeral es la habitual (3-1-3-3), siendo diminutas las setas de los pares $1 a, 1 c, 2 a$ y $3 a$, mientras que las restantes son más bien cortas y barbuladas, situándose las $4 c$ sobre el borde de unos puntiagudos discidios. La placa ventral presenta un amplio reticulado constituido por foveolas de contorno más o menos circular. Las placas genitales, que distan mucho de las anales (más del doble de su longitud), muestran cuatro pares de setas dispuestas en su borde interno; son de longitud similar a las epimerales y también están barbuladas; cada placa genital presenta un tenue e irregular surco longitudinal mediano; las setas adgenitales, similares a las genitales, están bastante distantes del borde posterior de las placas genitales. Las placas anales, en cambio, están densa- y nítidamente foveoladas y los dos pares de setas que portan son muy cortas y lisas, $y$ se disponen muy próximas al borde interno de cada placa; los tres pares de setas adanales son de aspecto más similar a las notogastrales, aunque más cortas, lanceolado-foliáceas (y también cubiertas de microespículas), estando las $a d 1$ en posición cláramente postanal y las $a d 3$ también cláramente preanales, localizándose las fisuras iad (cortas y difíciles de apreciar) muy distantes de los bordes laterales anteriores de las placas anales y en posición casi transversal.

PATAS: Son monodáctilas y presentan unas destacadas quillas ventrales en los trocánteres y fémures del tercer y cuarto par de patas; están adaptadas para replegarse en las oquedades existentes a los lados del cuerpo, en caso de necesidad, para su defensa.

AfINIDADES: Pertenecen al grupo de especies que Baratti y Bernini (1994) denominan "curtiseta". Por su sensilo corto y por el tamaño y forma de las setas notogastrales a la especie que más se parece la nueva es a Odontocepheus oglasae Baratti y Bernini, 1994 (a la que se asemeja mucho la especie descrita posteriormente de China Odontocepheus beijingensis Wang, 1997), pero se diferencia nítidamente de ella por el reticulado notogastral ya que dicha especie presenta foveolas que, aunque irregulares, son todas similares entre sí y más o menos redondeadas, pero nunca transversalmente rectangulares como presenta la nueva especie en la parte central del notogáster; además los dos pares de setas notogastrales anteriores, las $c 1$ y $c 2$, de $O$. zaballos $i$ son diferentes a las demás, más largas y en forma de gruesa cerda, no lanceolado-foliáceas como las restantes setas notogastrales, como es el caso de $O$. oglasae.

Biología: Su presencia en el suelo de un matorral típicamente mediterráneo, como es la Pistacea, nos está indicando, como era el caso de la especie anterior, sus tendencias xerófilas.

ETIMOLOGía: El nombre dado a la nueva especie de $O$. zaballosi está dedicado a don Juan P. Zaballos, amigo y prestigioso coleopterólogo, recolector de numerosas muestras de suelo, sobre todo del medio subterráneo superficial (MSS), que tan interesantes especies de oribátidos nos ha suministrado. 


\section{Referencias}

BARATti, M. \& Bernini, F., 1994. Odontocepheus oglasae n. sp. (Acari, Oribatida, Carabodidae) from Montecristo Island (Tuscan Archipelago). International Journal of Acarology, 20: 2537.

Baratti, M. \& Bernini, F., 1996. Notulae Oribatologicae LXIII. New reports of Carabodes chirstlus Mahunka, 1987 in the Western Mediterranean and the synonymization of $C$. foliatus Morell, 1990. Bollettino del Museo Civico di Storia Naturale de Verona, [1993], 20: 437-451.

Bernini, F., 1979. Notulae Oribatologicae XIX. Due nuove specie del genere Carabodes, $C$. quadrangulus e C. montanus (Acarida, Oribatida). Bollettino del Laboratorio di Entomologia Agraria "Filippo Silvestri", 36: 9-30.

Kunst, M., 1961. Bulgarische Oribatiden IV (Acari: Oribatei). Acta Universitatis Carolinae. Biologica, 2: 151-183.

Minelcic, F., 1966. Eine neue Oribatiden aus xerothermen Boden Zentralspaniens (Acarina). Eos, 42: 517 525.

Moraza, M. L., Herrera, L. \& PÉrrez-ÍÑIGO, C., 1980. Estudio faunístico del macizo de Quinto Real I: Ácaros Oribátidos (Acari, Oribatei). Publicaciones de Biología de la Universidad de Navarra, 1: 1-24.
Pérez-ÍñIgo, C., 1997. Acari. Oribatei, Gymnonota I. En: Ramos, M. A. et al. (eds.). Fauna Ibérica, 9. Museo Nacional de Ciencias Naturales, CSIC. Madrid. 373 pp.

Subías, L. S., Arillo, A. \& Gil-Martín, J., 1994. Oribátidos de Marruecos y Sahara Occidental II. Listado de especies (Acari, Oribatida). Boletín de la real Sociedad Española de Historia Natural (Sección Biológica), 91: 129-134.

Subías, L. S. \& Gil-Martín, J., 1997. Systematic and biogeographic checklist of Oribatids from Western Mediterranean (Acari, Oribatida). Annali del Museo Civico di Storia Naturale "Giacomo Doria", 91: 459-498.
Recibido, el 12-II-2001 Aceptado, 8-VI-2001 Publicado, el 20-VIII-2001 
